OPEN ACCESS

Edited by:

Daniela Carnevale,

Sapienza University of Rome, Italy

Reviewed by:

Giuseppe Faraco,

Weill Cornell Medicine, United States

Shinya Dohgu,

Fukuoka University, Japan

*Correspondence:

Guo-Yuan Yang

gyyang@sjtu.edu.cn

Zhijun Zhang

zhangzj@sjtu.edu.cn

Specialty section:

This article was submitted to

Non-Neuronal Cells,

a section of the journal

Frontiers in Cellular Neuroscience

Received: 29 September 2020

Accepted: 23 November 2020

Published: 12 January 2021

Citation:

Mamtilahun M, Wei Z, Qin C, Wang Y,

Tang $Y$, Shen F- $x$, Tian $H-L$, Zhang $Z$ and Yang G-Y (2021)

DL-3n-Butylphthalide Improves

Blood-Brain Barrier Integrity in Rat

After Middle Cerebral Artery

Occlusion

Front. Cell. Neurosci. 14:610714.

doi: 10.3389/fncel.2020.610714

\section{DL-3n-Butylphthalide Improves Blood-Brain Barrier Integrity in Rat After Middle Cerebral Artery Occlusion}

\author{
Muyassar Mamtilahun ${ }^{1}$, Zhenyu Wei ${ }^{2}$, Chuan Qin ${ }^{1}$, Yongting Wang ${ }^{1}$, Yaohui Tang ${ }^{1}$, \\ Fan-xia Shen ${ }^{3}$, Heng-Li Tian ${ }^{4}$, Zhijun Zhang ${ }^{1 *}$ and Guo-Yuan Yang ${ }^{1,3 *}$ \\ ${ }^{1}$ Shanghai Jiao Tong University Affiliated Sixth People's Hospital, School of Biomedical Engineering, Shanghai Jiao Tong \\ University, Shanghai, China, ${ }^{2}$ University of Shanghai for Science and Technology Affiliated Shidong Hospital, Shanghai, \\ China, ${ }^{3}$ Department of Neurology, Ruijin Hospital, School of Medicine, Shanghai Jiao Tong University, Shanghai, China, \\ ${ }^{4}$ Department of Neurosurgery, Shanghai Jiao Tong University Affiliated Sixth People's Hospital, Shanghai Jiao Tong \\ University, Shanghai, China
}

Objective: DL-3n-butylphthalide (NBP) has beneficial effects in different stages of ischemic stroke. Our previous studies have demonstrated that NBP promoted angiogenesis in the perifocal region of the ischemic brain. However, the molecular mechanism of NBP for blood-brain barrier protection in acute ischemic stroke was unclear. Here, we explored the neuroprotective effects of NBP on blood-brain barrier integrity in the acute phase of ischemic stroke in a rat model.

Methods: Adult male Sprague-Dawley rats $(n=82)$ underwent $2 \mathrm{~h}$ of transient middle cerebral artery occlusion and received $90 \mathrm{mg} / \mathrm{kg}$ of NBP for 3 days. Brain edema, infarct volume, surface blood flow, and neurological severity score were evaluated. Blood-brain barrier integrity was evaluated by Evans blue leakage and changes in tight junction proteins. We further examined AQP4 and eNOS expression, MMP-9 enzyme activity, and possible signaling pathways for the role of NBP after ischemic stroke.

Results: NBP treatment significantly increased eNOS expression and surface blood flow in the brain, reduced brain edema and infarct volume, and improved neurological severity score compared to the control group $(p<0.05)$. Furthermore, NBP attenuated Evans blue and IgG leakage and increased tight junction protein expression compared to the control after 1 and 3 days of ischemic stroke $(p<0.05)$. Finally, NBP decreased AQP4 expression, MMP-9 enzyme activity, and increased MAPK expression during acute ischemic stroke.

Conclusion: NBP protected blood-brain barrier integrity and attenuated brain injury in the acute phase of ischemic stroke by decreasing AQP4 expression and MMP-9 enzyme activity. The MAPK signaling pathway may be associated in this process.

Keywords: AQP4, blood-brain barrier, dl-3n-butylphthalide, edema, ischemic stroke 


\section{INTRODUCTION}

Stroke is one of the leading causes of death and disability worldwide (Feigin et al., 2017). Due to a narrow treatment window, complex pathology, and limited treatment options, stroke ultimately causes patient disability or death, proving to be an enormous economic burden to families and society (Writing Group et al., 2016; Feigin et al., 2017). Approximately $87 \%$ of stroke cases are ischemic strokes in the clinic; from the moment cerebral vascular occlusion occurs, the brain tissue suffers a series of pathological cascades including energy failure, increase in reactive oxygen species (ROS), free radical formation, inflammation, and neuronal apoptosis, which could last for several days (Kalogeris et al., 2016). These pathological processes upregulate aquaporin 4 (AQP4) expression and enhance matrix metalloproteinase-9 (MMP-9) enzyme activity. Both AQP4 and MMP-9 increase water uptake and degradation of protein in the blood-brain barrier (BBB) tight junction, which is the main cause of brain edema (Ribeiro et al., 2006; Lakhan et al., 2013; Turner and Sharp, 2016). Brain edema leads to cerebral parenchymal swelling, increased intracranial pressure, and decreased blood flow (Rosenberg, 1999; Mamtilahun et al., 2019). Without medical treatment, edema can result in secondary brain injury and aggravate stroke prognosis.

In the normal brain, the $\mathrm{BBB}$ facilitates brain homeostasis by separating the brain tissues from the peripheral circulation. $\mathrm{BBB}$ disruption is a canonical pathological characteristic in the acute stage of stroke. It not only causes brain edema but also allows the passage of phagocytes, red blood cells, and metabolic products that induce inflammatory responses and neuronal cell death, which together contribute to the high mortality of ischemic stroke (Yang and Rosenberg Gary, 2011; Obermeier et al., 2013). Hence, targeting $\mathrm{BBB}$ protection may be a viable approach for acute ischemic stroke therapy.

Dl-3n-butylphthalide (NBP) is a synthesized drug that was first extracted from Apium graveolens Linn seeds (celery) and approved for clinical usage in China by the National Medical Products Administration of China in 2002 (Wang et al., 2018). Clinical and experimental studies have shown that NBP attenuates cerebral infarct size and neurobehavioral deficiency through multitargeted effects, including antiplatelet aggregation (Wang et al., 2016), improvement of mitochondrial functions, antithrombosis (Qin et al., 2019), and reduction of both neurovascular inflammation (Yang et al., 2019) and apoptosis (Zhang et al., 2010). Previously, we found that NBP treatment promoted middle cerebral artery (MCA) dilation and increased microvessel density in ischemic rats (Qin et al., 2019; Zhou et al., 2019). NBP was approved for acute ischemic stroke stage II clinical studies by the Food and Drug Administration (FDA) in 2016 (Wang et al., 2018). Even though there are extensive studies on the therapeutic effects of NBP in ischemic stroke, more

Abbreviations: AQP4, Aquaporin4; BBB, blood-brain barrier; EB, Evans blue; eNOS, endothelial nitric oxide synthase; LSCI, Laser speckle contrast imaging; MCAO, middle cerebral artery occlusion; MMP-9, matrix metalloproteinase-9; NBP, dl-3n-butylphthalide; mNSS, modified neurological severity scores; ROS, reactive oxygen species; CBF, cerebral blood flow; PFA, paraformaldehyde; ZO-1, zonula occludens-1. robust evidence regarding its effects and underlying mechanisms is required to recommend universal clinical use. Particularly, the neuroprotective effects of NBP on BBB integrity and its underlying mechanism remain unclear. In the present study, we explored the effect of NBP on the functional and structural integrity of the BBB, and whether AQP4 and MMP-9 are involved in this process.

\section{MATERIALS AND METHODS}

\section{Experimental Design}

The rat brain surgery procedure and experimental protocol were approved by the Institutional Animal Care and Use Committee (IACUC) of Shanghai Jiao Tong University, Shanghai, China. Adult male Sprague-Dawley (SD) rats ( $n=82$, Jiesijie Laboratory Animal Co., Shanghai, China), weighing 250-280 g, were used in this study. All rats were kept in a humidity-controlled house at $22-25^{\circ} \mathrm{C}$ with 12 -h dark/light cycling, and were allowed to eat and drink freely. The SD rats underwent $2 \mathrm{~h}$ of transient middle cerebral artery occlusion (tMCAO) and were randomly assigned to NBP or vehicle (vegetable oil) treatment by daily oral gavage for 3 consecutive days. Cerebral blood flow was assessed using laser speckle contrast imaging (LSCI) at the five indicated time points (Figure 1A). Neurobehavioral outcomes of tMCAO rats were evaluated by neurological severity score (NSS) on days 1 and 3 after tMCAO, following which, rats were euthanized for further immunohistochemistry analysis. For Western blot and Evans blue analysis, the samples were collected from the indicated parts of the brain (Figure 1B). For immunostaining, five to seven animals in each group, with four brain sections from each animal were used. Next, four peri-infarct areas of each brain section were randomly chosen to take confocal images (Figure 1C).

\section{Surgical Procedure for Transient Middle Cerebral Artery Occlusion}

Ischemic brain injury was induced by $\mathrm{tMCAO}$, as described in our previous studies (Li et al., 2013). Briefly, the rat was anesthetized with $4 \%$ isoflurane, and anesthesia was maintained with $2 \%$ isoflurane during the entire surgical procedure. The body temperature was maintained at $37 \pm 0.5^{\circ} \mathrm{C}$ using a heating pad (RWD Life Science Co., Shenzhen, China). The left common carotid, internal carotid, and external carotid arteries were carefully isolated. A silicon coded 4-0 round top nylon suture (Covidien, Mansfield, MA, USA) was carefully inserted from the left external carotid artery into the internal carotid artery and the origin of the middle cerebral artery. The surface cerebral blood flow (CBF) was monitored, and a decrease to $80 \%$ of the baseline value was considered as MCA occlusion. Reperfusion was performed using a suture draw after $2 \mathrm{~h}$ of MCAO. To ensure reproducibility of the tMCAO model, the surface blood flow was monitored before and after $\mathrm{MCAO}$, and after reperfusion. Blood oxygen $\left(\mathrm{PO}_{2}\right)$, blood carbon dioxide $\left(\mathrm{PCO}_{2}\right)$, blood $\mathrm{pH}$, glucose (Glu), sodium $(\mathrm{Na})$, and potassium $(\mathrm{K})$ were determined using an i-STAT system (Abbott Point of Care Inc., Princeton, NJ, USA). NBP (purity $>99.5 \%$ ) was obtained as a gift from Shijiazhuang Pharmaceutical Group Co. Ltd., China, and dissolved in vegetable oil. tMCAO rats were randomly divided into two groups: NBP 
A (Fig. 2 and Fig. 3A)

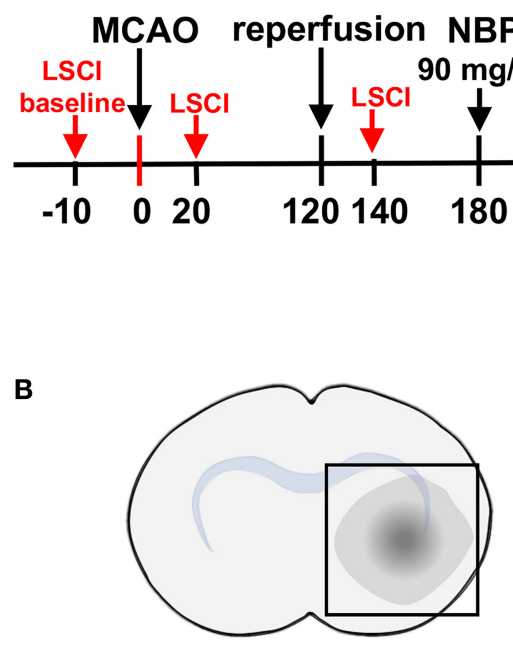

(Fig. 3C, Fig. 4A, Fig. 6, Fig. 7)

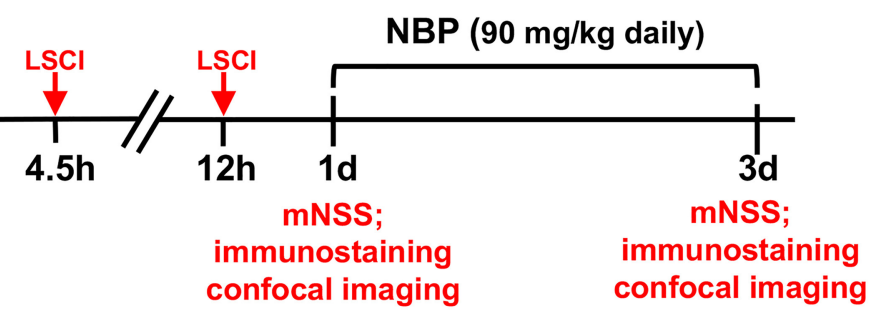

C

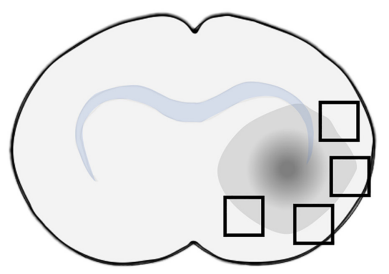

(Fig. 4C, Fig. 5)

FIGURE 1 | Schematic for experimental design and brain regions used for immunofluorescence analysis. (A) Graph illustrates the experimental design and sample collection schedule. Male SD rats underwent transient middle cerebral artery occlusion (tMCAO) for $2 \mathrm{~h}$; $90 \mathrm{mg} / \mathrm{kg}$ of dl-3n-butylphthalide (NBP) or vehicle was orally administered at $3 \mathrm{~h}$ after MCAO (1 h after reperfusion) for the first time, and then twice daily. The modified neurological severity scores (mNSS) scores were evaluated on day 1 and day 3 after tMCAO. (B) Photographs illustrate the brain samples collected after the neurobehavioral tests to evaluate gap formation in the blood-brain barrier (BBB) tight junction proteins. (C) Photographs illustrate brain regions used for immunostaining and analysis.

treated and control group. NBP $(90 \mathrm{mg} / \mathrm{kg}$ ) or equal volume of vegetable oil was orally administered $3 \mathrm{~h}$ after MCAO for the firsttime treatment, and then twice daily until sacrificing the animals. MAPK signaling inhibitor PD98059 (10 mg/kg, Meilun, Dalian, China) were tail intravenously injected to rats $1 \mathrm{~h}$ before tMCAO as per manufacturers' instruction.

\section{Evaluation of Neurological Severity Score}

At 1 and 3 days after tMCAO, modified NSS (mNSS) was examined to evaluate animal neurological status including sensory, motor, reflex, and balance tests (Tang et al., 2014). The normal mNSS was 0 , and the maximal deficit score was 14 ( $\mathrm{Li}$ et al., 2000). Rats were raised by the tail, and the flexion of the forelimb was tested for motor function evaluation (0-3). Rats' gait was examined by placing them on the floor (0-3). For the beam balance test, rats were placed on a $60-\mathrm{cm}$-long beam, and their posture was examined (0-6). The sensory tests (0-2) for touch-reflex were used to assess the pinna and corneal reflex.

\section{Laser Speckle Contrast Imaging}

LSCI was performed using a high-resolution laser speckle contrast imaging system (LSCI-2 system, Dolphin BioTech Ltd., Shanghai, China). The imaging protocol has been described previously (Lin et al., 2013). After anesthetizing the rats, a midline incision was made on the scalp, and the skull was exposed. A dental drill was used to remove the surface of the skull until surface blood vessels were detected beneath the skull. Raw speckle images $(696 \times 512$ pixels, $40 \mu \mathrm{m} /$ pixel $)$ were acquired at $23 \mathrm{fps}$ (exposure time $T=5 \mathrm{~ms}$ ) under $780 \mathrm{~nm}$ laser illumination. In each measurement, 20 consecutive frames of speckle images were detected and recorded. Image processing was carried out offline using MatLab software. In order to reduce the noise, the raw LSCI images were aligned using the registered laser speckle contrast analysis method (Wang et al., 2019). The registered speckle images were then analyzed using a random process estimator method to detect the contrast image with improved signal-to-noise ratio (Miao et al., 2010). Relative blood flow was calculated as described in our previous study (Lin et al., 2013). The CBF was detected using LSCI before MCAO, 10 min after occlusion, $20 \mathrm{~min}$ after reperfusion, and 4.5 and $12 \mathrm{~h}$ after occlusion in NBP-treated and control rats.

\section{Assessment of Brain Edema and Ischemic Infarct Volume}

Frozen brain sections $20 \mu \mathrm{m}$ in thickness from the anterior commissure to the hippocampus were collected for immunohistochemistry. Serial frozen sections, $20 \mu \mathrm{m}$ in thickness and $200 \mu \mathrm{m}$ in interval from the frontal cortex were stained with $0.1 \%$ cresyl violet (Meilun Chemical Reagent Co., Dalian, China). Infarct volume was determined by subtracting the area stained with cresyl violet in the ipsilateral hemisphere from that of the contralateral hemisphere using the Image J software (NIH, Bethesda, MD, USA, RRID: SCR_003070), and then multiplying by section interval thickness.

$$
v=\sum_{1}^{\mathbf{n}}\left[\left(\mathbf{s}_{\mathbf{n}}+\sqrt{\mathbf{s}_{\mathbf{n}} \times \mathbf{s}_{\mathbf{n}+1}}+\mathbf{s}_{\mathbf{n}+1}\right) \times \frac{\mathbf{h}}{3}\right]
$$

The $h=0.2 \mathrm{~mm}$ represents the distance between each section, and $S$ represents the area $\left(\mathrm{mm}^{2}\right)$ in each brain section. 


\section{Determining Permeability of the Blood-Brain Barrier}

Rats were sacrificed after 1 and 3 days of tMCAO using a high dose of chloral hydrate (10\%) anesthesia. The extravasation of Evans Blue (EB, Sigma-Aldrich, St. Louis, MO, USA) and IgG were used to assess $\mathrm{BBB}$ permeability. EB dye solution ( $2 \% \mathrm{~EB}$ dye in saline, $4 \mathrm{ml} / \mathrm{kg}$ ) was injected through the left jugular vein at $4.5 \mathrm{~h}, 1$ day, and 3 days after tMCAO (Tang et al., 2014). The rats were sacrificed via cardiac perfusion after $2 \mathrm{~h}$ of EB circulation under anesthetized conditions. Both brain hemispheres were weighed, and the samples were homogenized in $1 \mathrm{ml}$ of $50 \%$ trichloroacetic acid solution to extract EB, followed by centrifugation at $12,000 \mathrm{~g}$ for $20 \mathrm{~min}$. The supernatant was diluted with $100 \%$ ethanol at a ratio of 1:3. The amount of EB was quantified at $610 \mathrm{~nm}$ using a spectrophotometer (Bio-Tek, Winooski, VT).

IgG was examined as previously described (Tanno et al., 1992; Tang et al., 2014). Briefly, brain slices were fixed in $4 \%$ paraformaldehyde (PFA) solution, blocked in 10\% bovine serum albumin (BSA), incubated in biotinylated antibody for $30 \mathrm{~min}$ at room temperature, and incubated in Avidin: Biotinylated Enzyme Complex $(\mathrm{ABC})$ reagent (Vector Labs, Burlingame, CA, USA) for $30 \mathrm{~min}$. DAB staining was used for the visualization of immune reactivity (Vector Labs, Burlingame, CA, USA), and the sections were counterstained with hematoxylin. We randomly chose four fields from the area of interest in each section and used the Image J software (NIH, Bethesda, MD, RRID: SCR_003070) for mean integrated optical density (IOD) analysis.

\section{Immunohistochemistry}

Brain sections were fixed for $10 \mathrm{~min}$ in cold methanol at $4^{\circ} \mathrm{C}$, then blocked with $10 \%$ BSA for $1 \mathrm{~h}$ at room temperature after washing with PBS thrice. The brain sections were then incubated with antibodies against occludin (1:100, Invitrogen Cat\# 33-1500, RRID: AB_87033), zonula occludens-1 (ZO-1, 1:100, Invitrogen Cat\# 61-7300, RRID: AB_138452), and CD-31 (1:200, R\&D Systems Cat\# AF3628, RRID: AB_2161028) overnight at $4^{\circ} \mathrm{C}$. The sections were stained with fluorescence-conjugated secondary antibodies for $1 \mathrm{~h}$ at $37^{\circ} \mathrm{C}$ after washing with PBS thrice. The gap length was shown as a percentage (\%) of the whole tight junction staining. Similarly, four brain sections were stained for each animal, and four fields were randomly selected from each brain section (upper, middle, and bottom of the peri-infarct region), using confocal microscopy. Data were analyzed using the Image J software (NIH, Bethesda, MD, USA, RRID: SCR_003070).

\section{Western Blot Analysis}

At 1 and 3 days after tMCAO, fresh rat brains were collected and sectioned into 2-mm slices that included the ischemic core and peri-infarct areas in a rat brain mold (RWD Company, Shenzhen, China), and peri-infarct areas were collected from the ipsilateral cerebral hemisphere for the Western blotting experiments. Homogenizing buffer (RIPA with protease cocktail inhibitor, phosphatase inhibitor, and phenylmethanesulfonyl fluoride) was used for the brain sample collection. The homogenate was centrifuged at $12,000 \mathrm{~g}$, and the pellets were discarded. Protein concentration was examined using a BCA kit (Meilun, Dalian, China), and $40 \mu \mathrm{g}$ of protein from each group was loaded onto $10 \%$ resolving gel for electrophoresis. Then protein was transferred to a nitrocellulose membrane (GE Healthcare Life Sciences, Pittsburgh, PA, USA), and 5\% skim milk was used for blocking. Next, the membranes were incubated with primary antibodies against AQP4 (1:1,000, Santa Cruz Biotechnology Cat\# sc-58612, RRID:AB_781471), occludin (1:1,000, Invitrogen Cat\# 33-1500, RRID: AB_87033), zonula occludens-1 (ZO-1, 1:1,000, Invitrogen Cat\# 61-7300, RRID: AB_138452), eNOS (1:1,000, BD Biosciences Cat\# 610298, RRID:AB_397692), phospho-MAPK (1:1,000, Cell Signaling Technology Cat\# 4370, RRID:AB_2315112), MAPK (1:1,000, Cell Signaling Technology Cat\# 4695, RRID:AB_390779), and $\beta$-actin (1:1,000, Santa Cruz Biotechnology Cat\# sc-47778 HRP, RRID:AB_2714189) overnight at $4^{\circ} \mathrm{C}$. HRP-conjugated secondary antibody and enhanced chemiluminescence substrate (Pierce, Rockford, IL, USA, www.piercenet.com) were used for visualization after washing thrice with TBST buffer. The results of chemiluminescence were assessed with an imaging system (Bio-Rad, Hercules, CA, USA, www.bio-rad.com). The relative levels of AQP4, occludin, ZO-1, and eNOS were normalized to that of $\beta$-actin. The pMAPK levels were normalized to those of total MAPK.

TABLE 1 | Analysis of blood parameters.

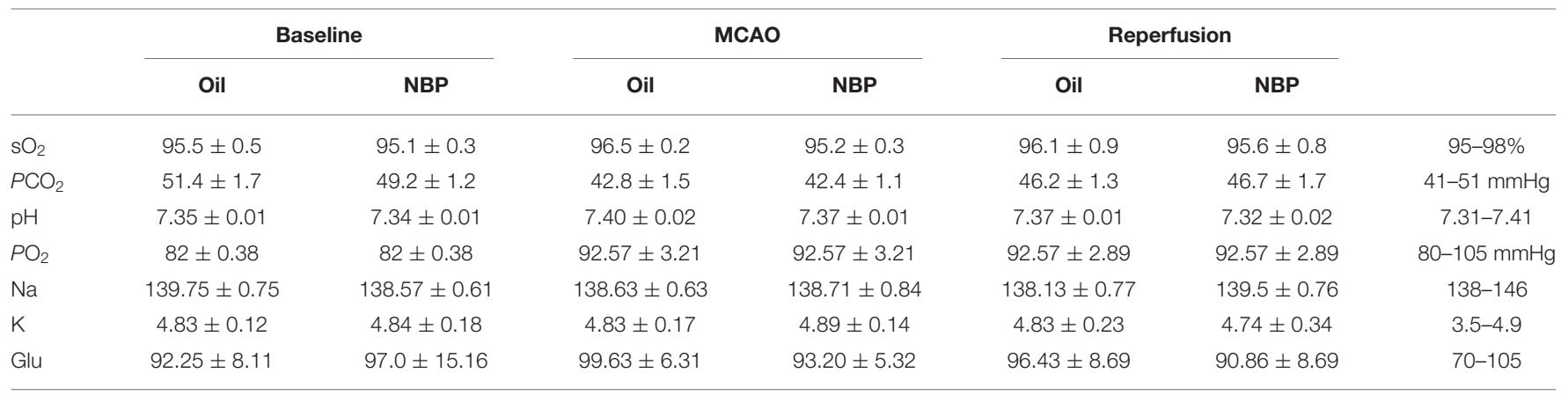

Blood parameters $\left(\mathrm{sO}_{2}, \mathrm{pO}_{2}, \mathrm{pCO}_{2}, \mathrm{~K}, \mathrm{Na}\right.$, Glu) before and during the occlusion, and after reperfusion. Data are mean $\pm \mathrm{SD} ; n=8$ per group. 


\section{Real-Time PCR Analysis}

Total RNA from brain tissue samples was isolated using TRIzol Reagent (Life Technologies). RNA concentration was examined using a spectrophotometer (NanoDrop 1000, Thermo Fisher) followed by a reverse transcription process using the PrimeScript RT reagent kit (TaKaRa, Dalian, China, www.takara.com.cn). SYBR Premix Ex Tag Kit (TaKaRa, Dalian, China) was used to perform real-time PCR. A two-stage RT-PCR amplification reaction was performed under the following conditions: $95^{\circ} \mathrm{C}$ for $30 \mathrm{~s}$, followed by 40 cycles at $95^{\circ} \mathrm{C}$ for $5 \mathrm{~s}$, and at $60^{\circ} \mathrm{C}$ for $30 \mathrm{~s}$. The primer sequences were: AQP4 forward primer: 5'-GGGTTGGACCAATCATAGG CG-3', reverse primer: 5' GCAGGAAATCTGAGGCCAGTTCTAGG-3'; MMP-9 forward primer: 5'-CGCTGACAAGAAGTGGGGTTT-3', reverse primer: 5'-TACAGATGGTGGATGCCTTTTA G-3'; GAPDH forward primer: $5^{\prime}$-TGAACGGGAAGCTCACTGG-3', reverse primer: 5'-GCTTCACCACCTTCTTGATGTC-3'.

\section{Gelatin Zymography}

Previous zymography protocol was followed here (Cai et al., 2015). Briefly, $50 \mu \mathrm{g}$ samples were diluted in the zymogram sample buffer (Bio-Rad) and electrophoresed using SDS-PAGE for $\sim 2.5 \mathrm{~h}$. Then the gels were incubated four times in renaturing buffer $(2.5 \%$ Triton X-100, $50 \mathrm{mmol} / \mathrm{L}$ Tris- $\mathrm{HCl})$ for $15 \mathrm{~min}$ each with gentle shaking, and then moved to developing buffer for 30 min with gentle shaking at room temperature. Next, gels were stained with Coomassie Blue $(0.05 \%$ Coomassie Brilliant Blue, $30 \%$ methanol, $10 \%$ acetic acid) for $3 \mathrm{~h}$ after incubation in fresh developing buffer for 3 days at $37^{\circ} \mathrm{C}$, and then $30 \%$ methanol containing $10 \%$ acetic acid was used for destaining to achieve proper color contrast. The final bands were quantified using the Image J software (NIH, Bethesda, MD, USA, RRID: SCR_003070).

\section{Statistical Analysis}

Data are shown as mean \pm SD. Statistical analysis was performed using SPSS and GraphPad PRISM 6.0 software (RRID: SCR_002798) for both parametric and non-parametric comparisons. Unpaired two-tailed Student's $t$-test (between two groups) and one-way ANOVA followed by StudentNewman-Keuls (among multiple groups) were used to evaluate statistical significance. Differences with $p<0.05$ were considered significant.

\section{RESULTS}

\section{NBP Attenuated Brain Edema, Increased Cerebral Blood Flow, and Improved Neurobehavioral Outcomes in Transient Middle Cerebral Artery Occlusion Rats}

Blood parameters including $\mathrm{pH}, \mathrm{PCO}_{2}, \mathrm{PO}_{2}$, sodium, potassium, and glucose concentration were measured before the surgery, 10 min after occlusion, and immediately after reperfusion. No significant changes in physiological parameters were detected
A

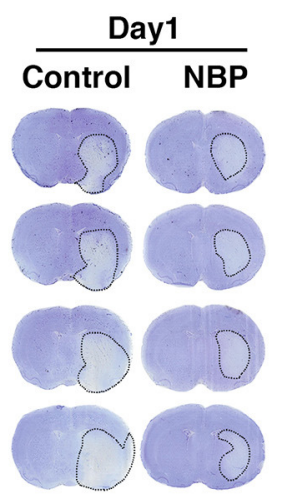

C

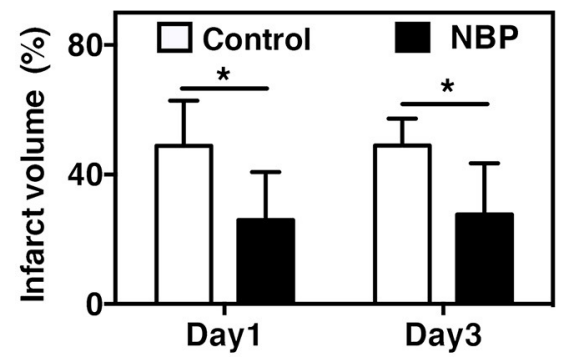

Day3
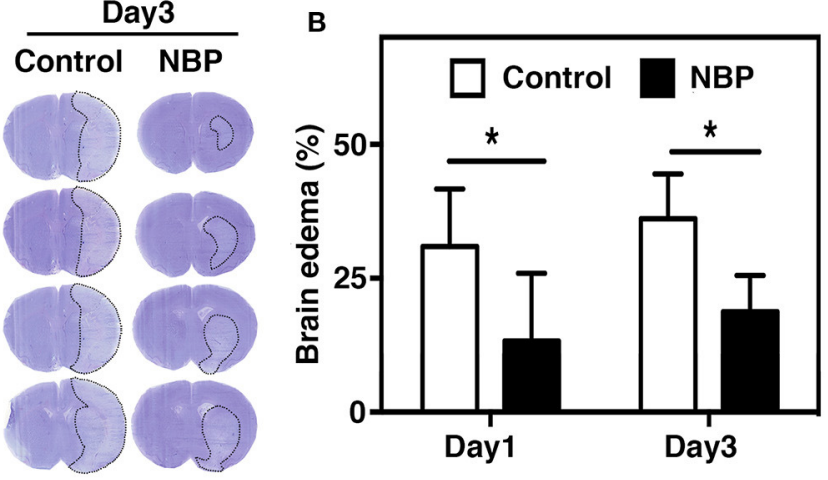

D

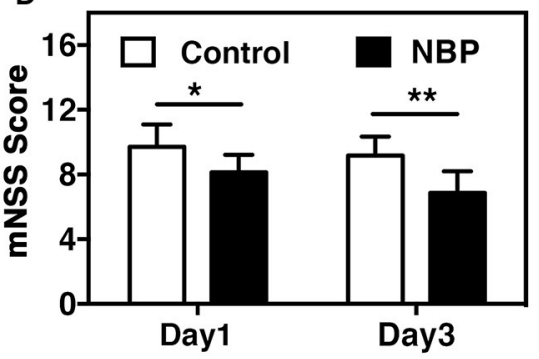

FIGURE 2 | NBP reduced edema and improved neurobehavioral outcomes in tMCAO rats. (A) Photomicrography represents the cresyl violet staining in NBP-treated and the control rats at 1 and 3 days after tMCAO. The dashed line indicates the border of the infarct area. Semi-quantification of the brain edema volume (B) and the infarct volume (C) in the NBP-treated and the control rats at 1 and 3 days after IMCAO. (D) Bar graph shows the mNSS assessment in the NBP-treated and the control rats at 1 and 3 days after tMCAO. Data are mean $\pm \mathrm{SD} ; n=8$ per group; ${ }^{*} p<0.05$ and ${ }^{* *} p<0.01$; NBP-treated rats vs. control rats. 
A

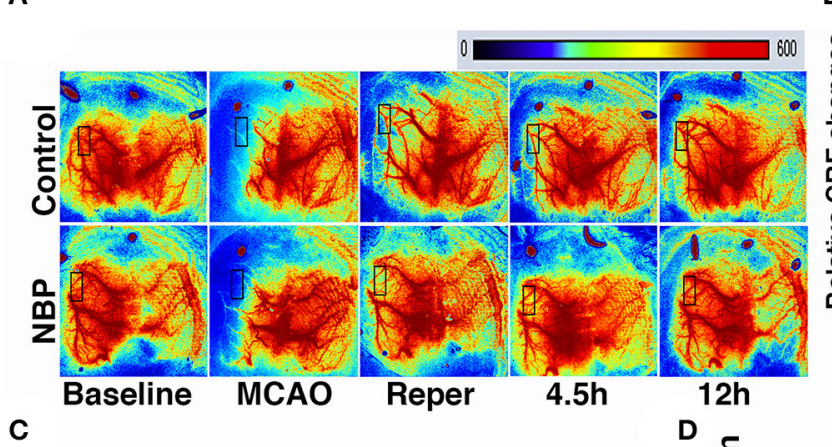

D
B

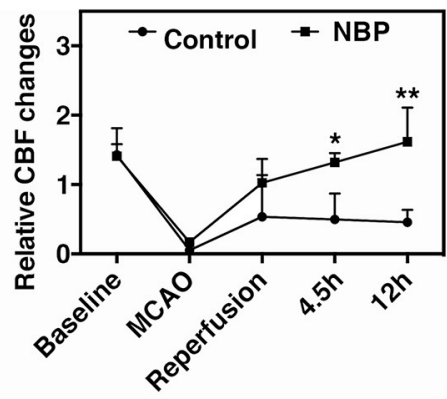

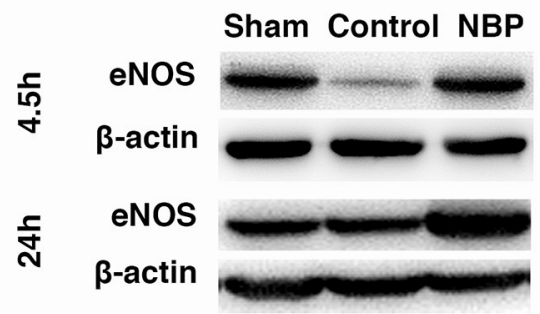

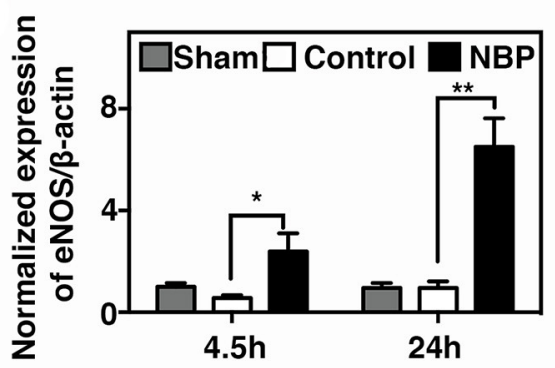

FIGURE 3 | NBP increased cerebral blood flow (CBF) in rats after tMCAO. (A) Photomicrographs show CBF measured by Laser speckle contrast imaging (LSCl) at the baseline, $10 \mathrm{~min}$ after MCAO, 10 min after reperfusion, and 4.5 and $12 \mathrm{~h}$ after occlusion in NBP-treated and control animals. (B) Bar graph shows the change in surface CBF in the ipsilateral hemisphere at different time points. Data are mean \pm SD; $n=5$ per group; ${ }^{*}<0.05$ and ${ }^{* *} p<0.01$; NBP-treated group vs. control animals. Bar graphs show Western blot images (C) and eNOS protein expression (D) in the NBP-treated and the control rats at 2.5 and $24 \mathrm{~h}$ after reperfusion. Data are mean $\pm \mathrm{SD} ; n=3$ per group; ${ }^{\star} p<0.05$ and ${ }^{* \star} p<0.01$; NBP-treated group vs. control animals.

before and after tMCAO operation (Table 1). Since ischemiainduced BBB disruption could lead to brain edema, we measured brain edema and brain infarct volume in NBP-treated rats at 1 and 3 days after tMCAO. The results showed that the ischemic infarct area decreased in the NBP-treated rats compared to that of the control (Figure 2A). It was noted that the infarct area was limited to the striatum in the NBP-treated rats, while it was larger in both the cortex and striatum of control rats. NBP treatment also decreased the brain edema volume and infarct volume (Figures 2B,C) compared to the control after 1 and 3 days of tMCAO $(p<0.05)$. Meanwhile, the mNSS evaluation showed that NBP treatment reduced neurobehavioral deficiency after ischemic brain injury both at 1 and 3 days of tMCAO $(p<$ 0.01 , Figure 2D).

A laser speckle contrast imaging system was used to monitor $\mathrm{CBF}$ during $\mathrm{tMCAO}$ surgery and assess the $\mathrm{CBF}$ dynamic changes after NBP treatment. The results showed that NBP treatment significantly increased CBF after reperfusion $(p<0.05)$ and maintained the increase until $12 \mathrm{~h}$ after tMCAO $(p<0.01)$. Meanwhile, the control group showed a decreasing tendency in $\mathrm{CBF}$ changes after reperfusion (Figures 3A,B). To further explore which molecular mechanism induces increase in $\mathrm{CBF}$, we examined endothelial nitric oxide synthase (eNOS) expression due to its role in vasodilation (Coletta et al., 2012). Our results showed that NBP treatment increased eNOS protein expression compared to the control at 4.5 and $24 \mathrm{~h}$ after occlusion (Figures 3C,D, $p<0.05$ ), which could explain why $\mathrm{CBF}$ increased after NBP treatment.

\section{DL-3n-Butylphthalide Protected Blood-Brain Barrier Integrity After} Transient Middle Cerebral Artery Occlusion To evaluate $\mathrm{BBB}$ functional integrity, we injected $2 \%$ Evans blue (EB) in the rats using the jugular vein on $4.5 \mathrm{~h}$, day 1 , and day 3 after tMCAO. EB extravasation was limited to the striatum of the ipsilateral hemisphere after NBP treatment, while EB extravasation was detected in both the striatum and cortex in the control group (Figure 4A), which was consistent with the cresyl violet staining results (Figure 2A). The quantification of EB results showed that the total amount of extravasated EB decreased in NBP-treated rats compared to the control at 1 and 3 days after tMCAO ( $p<0.01, p<0.001$, Figure 4B). To further evaluate $\mathrm{BBB}$ permeability, we stained the brain sections with the IgG antibody to assess the severity of the IgG invasion at 1 and 3 days after tMCAO. Results showed that IgG invasion mainly occurred in the striatum of NBP-treated rat brains, while both striatum and cortex showed IgG invasion in the control (Figure 4C). Semiquantification analyses showed that NBP treatment decreased IgG invasion compared to the control at 1 and 3 days after tMCAO ( $p<0.01, p<0.05$, Figure 4D). Furthermore, to investigate whether NBP protected BBB structural integrity, 

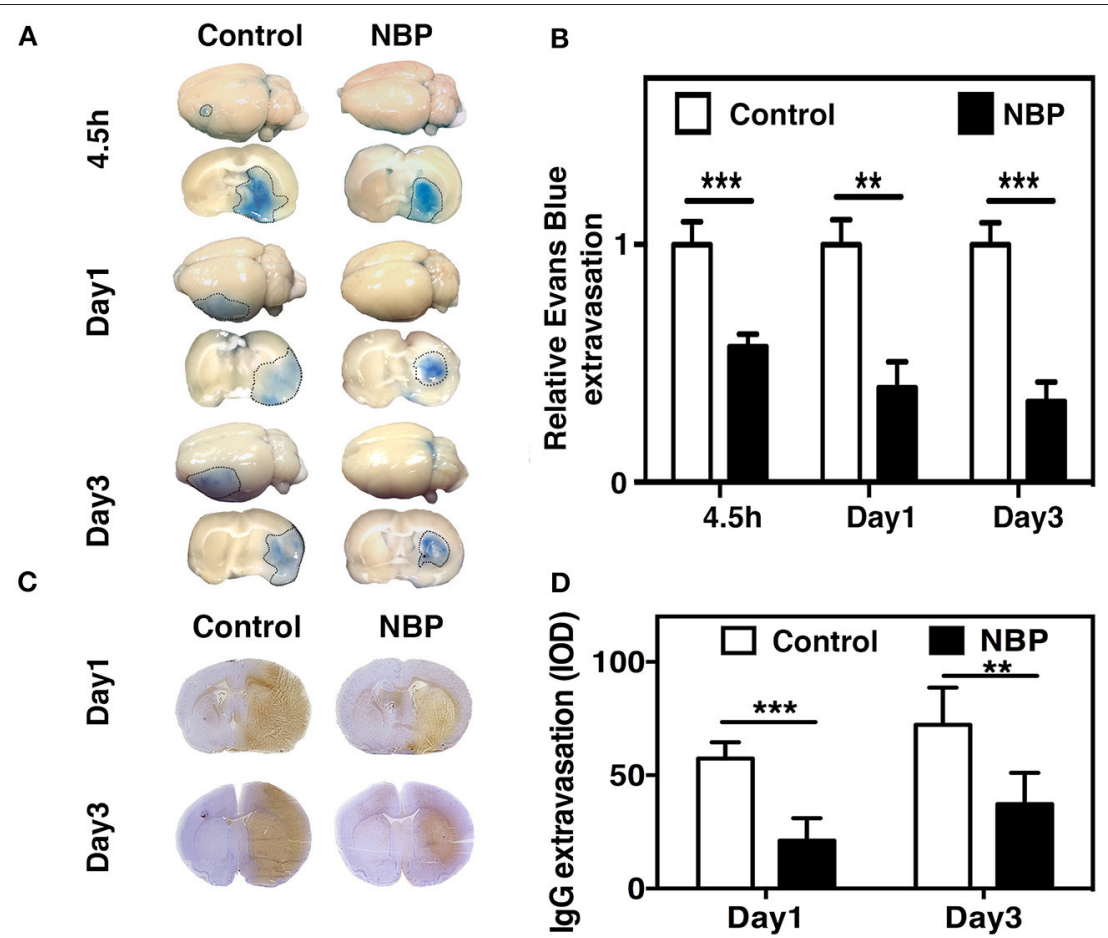

FIGURE 4 | NBP reduced BBB leakage in rats after TMCAO. (A) Photographs shows Evans blue (EB) exudation for the whole brain and brain sections in the NBP-treated and control rats at 1 and 3 days after tMCAO. The value of extravasated EB was recorded by a spectrophotometer at $610 \mathrm{~nm}(\mathrm{~g})$. (B) Bar graph shows semi-quantification for the relative EB extravasation in the NBP-treated and the control rats at $4.5 \mathrm{~h}, 1$ day, and 3 days after tMCAO. Data are mean $\pm \mathrm{SD} ; n=8$ per group; ${ }^{* \star} p<0.01$ and ${ }^{\star \star *} p<0.001$; NBP-treated rats vs. control rats. (C) Photographs show the lgG leakage in the ipsilateral hemisphere of NBP-treated and control rats following 1 and 3 days after tMCAO. (D) Bar graph shows semi-quantification of lgG extravasation in the NBP-treated and control rats. Data are mean \pm SD; $n=$ 7 per group; ${ }^{* *} p<0.01$, and ${ }^{* * *} p<0.001 ;$ NBP-treated rats vs. the control rats.

we stained the tight junction proteins $\mathrm{ZO}-1$ and occludin with endothelial cell marker CD31. The confocal microscopy images showed that NBP treatment significantly attenuated ZO1 and occludin-mediated gap formation in the microvessel wall induced by stroke, thereby preserving tight junction protein structural integrity at 1 and 3 days after tMCAO (Figures 5A-D).

\section{DL-3n-Butylphthalide Preserved Blood-Brain Barrier Integrity by Decreasing AQP4 Expression and MMP-9 Activity After Transient Middle Cerebral Artery Occlusion}

AQP4 overexpression and disruption of ionic balance contribute to brain edema after ischemic brain injury (Yang et al., 2007; Kleffner et al., 2008). Our results demonstrated that NBP could downregulate AQP4 expression at both the protein $(p<0.01, p$ $<0.05$, Figures 6A-D) and mRNA levels ( $p<0.01$, Figure 6E). Occludin expression increased in NBP-treated rats at 1 and 3 days after tMCAO, while ZO-1 expression only increased at 3 days after tMCAO (Figures 6A-D, $p<0.05$ ).

MMP-9 mediates BBB permeability by degrading tight junction proteins in the acute stage of stroke (Yang et al., 2007; Rosell et al., 2008). We next examined whether NBP had a protective effect on BBB permeability by reducing MMP-9 activity after ischemic brain injury. First, we found that MMP-9 at the mRNA levels decreased in the NBP-treated rats compared to the control after 1 and 3 days after tMCAO $(p<0.05$, Figure 6F). Second, to evaluate the MMP-9 enzyme activity, gelatin zymography was performed in the NBP-treated and control rats at 1 and 3 days after tMCAO. Results showed that MMP-9 activity was reduced in the NBP-treated rats compared to the control rats at 3 days after tMCAO $(p<0.01$, Figures 7A,B).

\section{MAPK Signaling Pathway Involved in the Protective Role of DL-3n-Butylphthalide After Transient Middle Cerebral Artery Occlusion}

To further explore the signaling pathway involved in the protective role of $\mathrm{NBP}$ on $\mathrm{BBB}$ integrity after $\mathrm{tMCAO}$, we examined the MAPK signaling pathway. The results showed that MAP kinase phosphorylation decreased in the NBP-treated rats compared to the control at 3 days after tMCAO (Figures 7C,D, $p<0.05)$. PD98059 inhibited MAPK phosphorylation in PD98059+NBP-treated group compared to the control group after tMCAO and diminished the effect of NBP on MAPK phosphorylation (Figures 7E,F, $p<0.05$ ). 


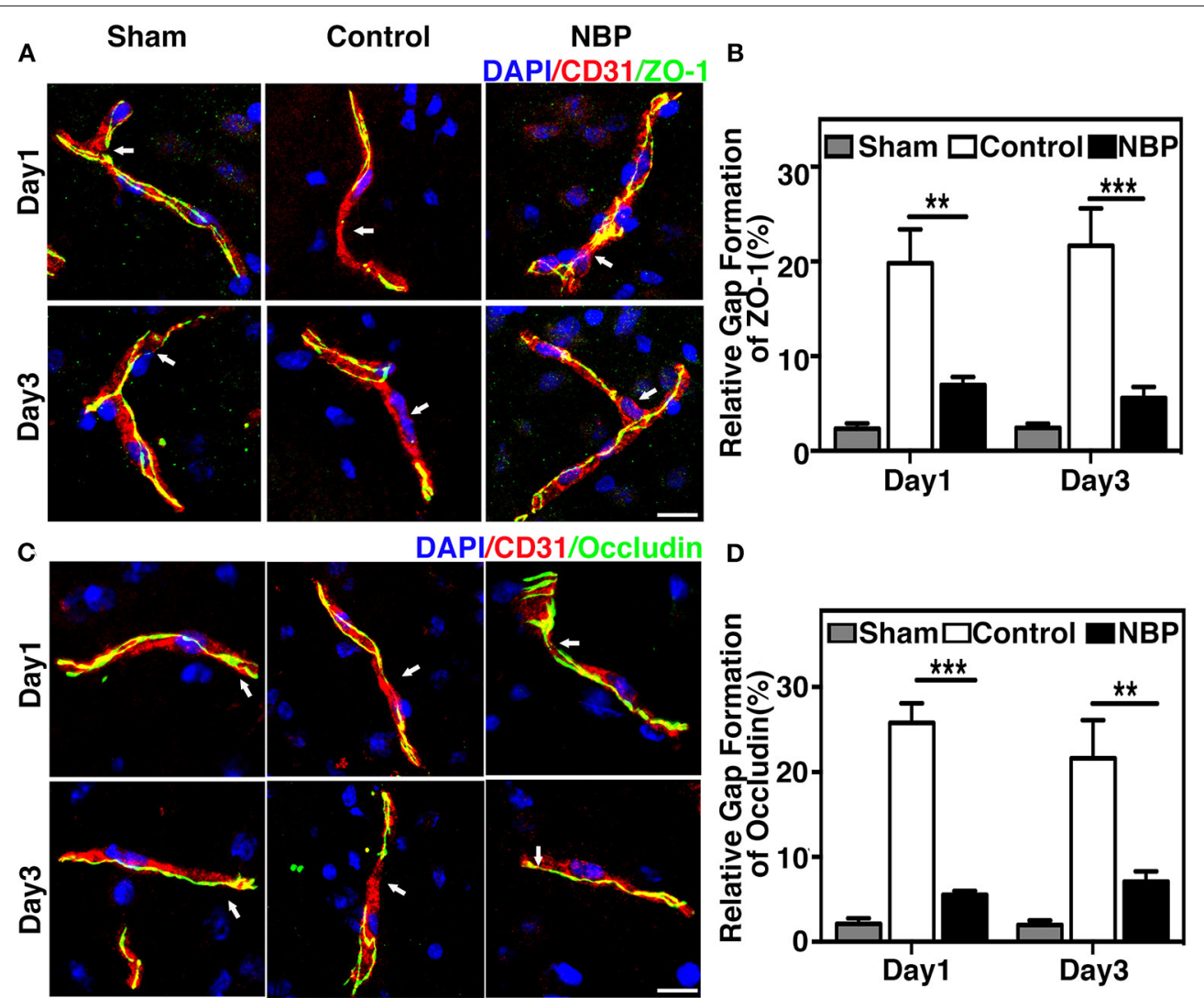

FIGURE 5 | NBP reduced BBB tight junction disruption in rats after tMCAO. (A) Photomicrographs show the ZO-1 (green) and CD31 (red) double immunostaining in the NBP treated and control rats at 1 and 3 days after tMCAO. Scale bar $=25 \mu \mathrm{m}$. (B) Bar graph shows the semi-quantification of gap length for ZO- 1 -positive staining. (C) Photomicrographs showed the occludin (green) and CD31 (red) double immunostaining in the NBP-treated and control rats at 1 and 3 days after tMCAO. Scale bar $=25 \mu \mathrm{m}$. (D) Bar graph shows the semi-quantification of gap length for occludin-positive staining. Data are mean $\pm \mathrm{SD} ; n=5$ per group; ${ }^{\star \star} p<0.01,{ }^{\star \star \star} p$ $<0.001$; NBP-treated rats vs. the control rats.

\section{DISCUSSION}

In the present study, we demonstrated that NBP (1) attenuated ischemia-induced brain edema and neuronal death; (2) improved neurological function recovery; (3) inhibited AQP4 expression in the ischemic brain and reduced tight junction protein loss; (4) and partially inhibited MMP-9 expression and activity (5) through the possible MAPK signaling pathway associated in BBB disruption (Figure 8). Our results provide experimental evidence that NBP improves the damaged $\mathrm{BBB}$ integrity during brain edema, suggesting that NBP could be used for BBB disruption induced by ischemia or other brain diseases.

Ischemic brain injury is common; it causes neuronal apoptosis, inflammatory response, free radical oxidants, and BBB disruption (Feigin et al., 2017). The disrupted BBB allows vasculature-derived substances to infiltrate the brain, induces secondary brain injury and edema, and aggravates ischemic stroke outcomes (Sandoval and Witt, 2008). We chose days 1 and 3 after tMCAO because BBB damage occurs in the early stage after stroke (Rosenberg, 1999; Khatri et al., 2012). We present the data at days 1 and 3 to interpret the effect of NBP on BBB integrity after ischemia. NBP has been used in the acute phase after ischemic stroke after several studies demonstrated its neuroprotective effects including dilation of blood vessels, promotion of angiogenesis, suppression of inflammation, antioxidative stress, protection of the structure and function of mitochondria, and inhibition of thrombosis (Abdoulaye and Guo, 2016; Qin et al., 2019; Yang et al., 2019; Zhou et al., 2019). NBP also attenuated ischemic brain injury by decreasing infarct volume, neuronal apoptosis, and neurological deficits in the peri-infarct areas in a rat disease model (Li et al., 2010; Zhang et al., 2012a,b). Clinical and experimental studies showed that as a lipid-soluble drug, NBP could directly pass through the $\mathrm{BBB}$ and exert its protective effects on the brain (Liu et al., 2007; Cao et al., 2009; Liao et al., 2009; Wang et al., 2011; Zhang et al., 2012a, 2017). In vitro study showed that NBP could protect BBB tight junction protein by decreasing the endothelial intracellular ROS generation (Ye et al., 2019). Our results demonstrated that NBP had protective effects on the $\mathrm{BBB}$ in an acute ischemic stroke animal model. The results showed that NBP reduced tight junction protein loss and AQP4 expression. Since the inhibition of AQP4 protein ameliorated 
A
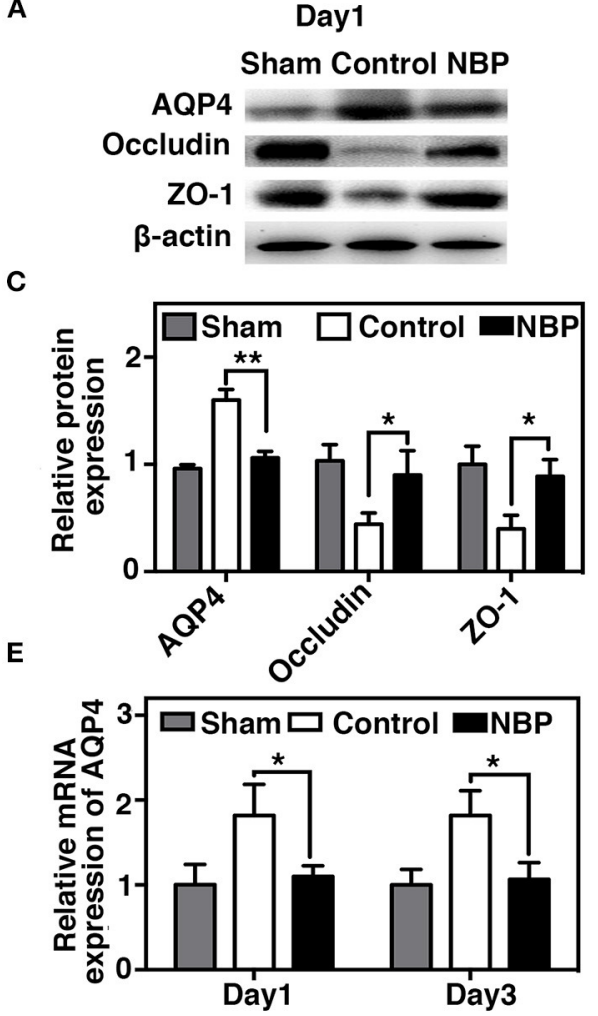

B

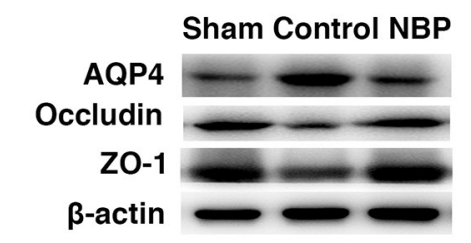

D

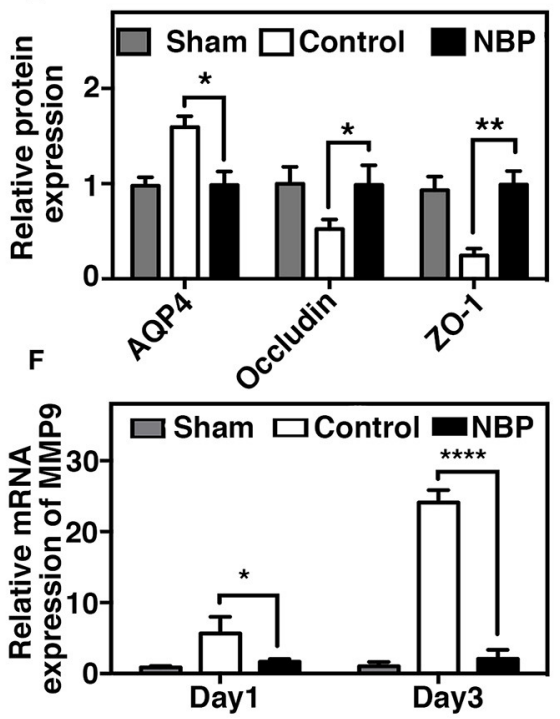

FIGURE 6 | NBP decreased AQP4 expression and tight junction protein loss after tMCAO. Photomicrographs showing the Western blot of AQP4, ZO-1, and occludin expression in the NBP-treated and control rats at 1 (A) and 3 days (B) after tMCAO. Bar graphs show the semi-quantification of AQP4, ZO-1, and occludin expression in the NBP-treated and control rats at 1 day (C) and 3 days (D) after tMCAO, and relative AQP4 (E) and matrix metalloproteinase-9 (MMP-9) (F) mRNA expression in the NBP-treated and control rats. Data are presented as mean $\pm \mathrm{SD} ; n=3$ per group; ${ }^{\star} p<0.05,{ }^{* \star} p<0.01,{ }^{\star \star \star *} p<0.0001 ;$ NBP-treated rats vs. control rats.

ischemic brain injury by reducing early cytotoxic brain edema, NBP may further protect the BBB from ischemia and reperfusion injury by downregulating AQP4. Preservation of the BBB tight junction proteins is essential for maintaining $\mathrm{BBB}$ integrity, which prevents secondary brain injury and is closely correlated with AQP4 downregulation (Tang et al., 2014; Filchenko et al., 2020). NBP has a therapeutic effect on the disrupted BBB in the early phase of ischemic stroke.

MMP-9 also plays a critical role in maintaining BBB integrity (Cai et al., 2015). Studies have shown that MMP-9 is not only involved in the pathogenesis of BBB disruption and subsequent vasogenic edema following stroke but also in hemorrhagic transformation (Lakhan et al., 2013; Shi et al., 2016). MMP9 upregulation results in the degradation of tight junction proteins such as ZO-1, occludin, and the basal lamina, ultimately triggering $\mathrm{BBB}$ disruption and brain edema in the acute stage of stroke (Yang et al., 2007; Lee et al., 2013; Mamtilahun et al., 2019). The inhibition of MMP-9 could reverse this effect (Wang et al., 2012). Here, we found that NBP could inhibit MMP-9 mRNA expression and MMP-9 enzyme activity. NBP-mediated MMP-9 activity downregulation could reduce $\mathrm{BBB}$ tight junction protein degradation and protect $\mathrm{BBB}$ integrity.
Additionally, we found that NBP increased MAP kinase phosphorylation. Studies have shown that MAP kinase ERK1/2 modulates the internalization and degradation of tight junction proteins claudin-2, claudin-4, occludin, and ZO-1 (RinconHeredia et al., 2014; Stamatovic et al., 2017). Phosphorylation of MAPK is usually induced by pro-inflammatory stimuli; it also can be activated by growth factor (Sun and Nan, 2016). In the present study, we found that NBP treatment modulated the MAPK phosphorylation to the same level as the sham group after tMCAO, and treating with MAPK inhibitor reversed the result. Previous studies showed that NBP treatment upregulates the VEGF and basic fibroblast growth factor (bFGF) expressions in ischemic stroke patients and improve neurobehavioral recovery in rat ischemic stroke model (Liao et al., 2009; Tang et al., 2017; Zhou et al., 2019); growth factor binds to its receptor to activate MAPK by B-Raf (Kao et al., 2001). Taken together, the increased phosphorylation of MAPK may be due to the upregulated growth factor after NBP treatment, which is closely related to angiogenesis and long-term recovery after stroke. Our results support that NBP protects $\mathrm{BBB}$ integrity by increasing the phosphorylation of MAP kinase pathway after stroke. 


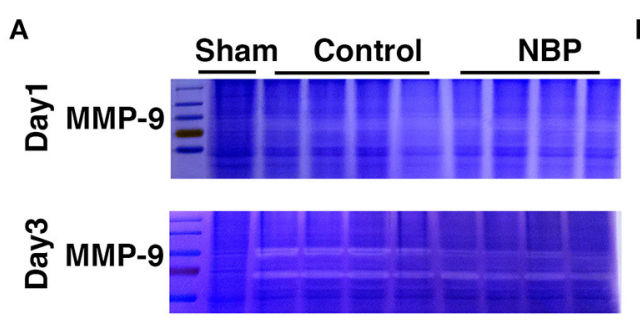

B

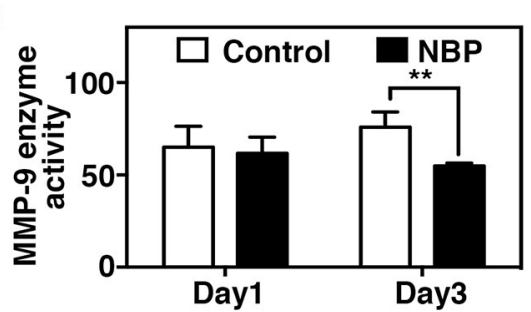

C

D
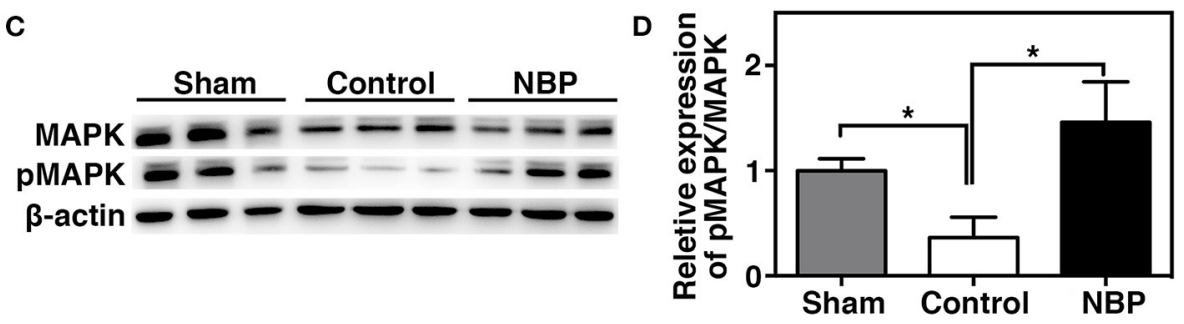

E
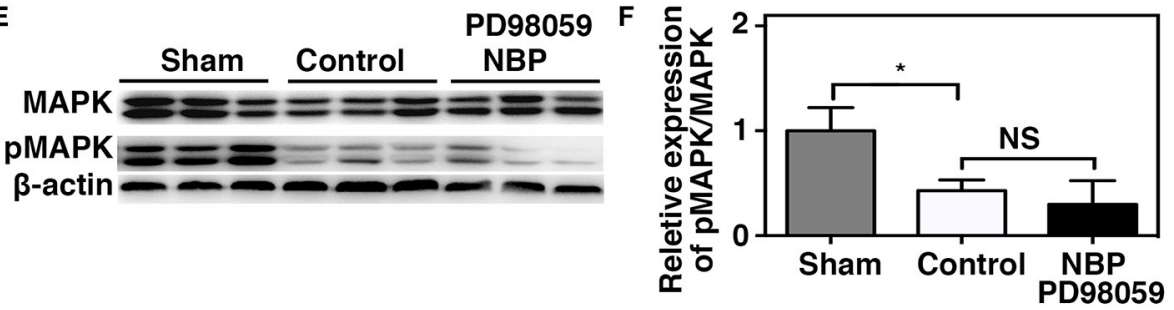

FIGURE 7 | NBP decreased MMP-9 enzyme activity, increased MAP kinases phosphorylation after tMCAO. (A) Photomicrograph shows zymography in NBP-treated and control rats at 1 and 3 days after tMCAO. (B) Bar graph shows the semi-quantification of active MMP-9 levels. Data are mean \pm SD; $n=4$ per group; ${ }^{* *} p<0.01$; NBP-treated rats vs. control rats. (C) Photomicrograph showed a Western blot of MAP kinases phosphorylation in the NBP-treated, control, and sham rats. (D) Bar graph shows semi-quantification of phospho-MAP kinase at 3 days after IMCAO. (E) Photomicrograph showed a Western blot of MAP kinases phosphorylation in the sham, Control, and PD98059+NBP-treated rats. (F) Bar graph shows semi-quantification of phospho-MAP kinase at 3 days after tMCAO. Data are mean \pm SD, $n=$ 3 per group; ${ }^{*} P<0.05,{ }^{* *} p<0.01$; NBP-treated rats vs. control rats.

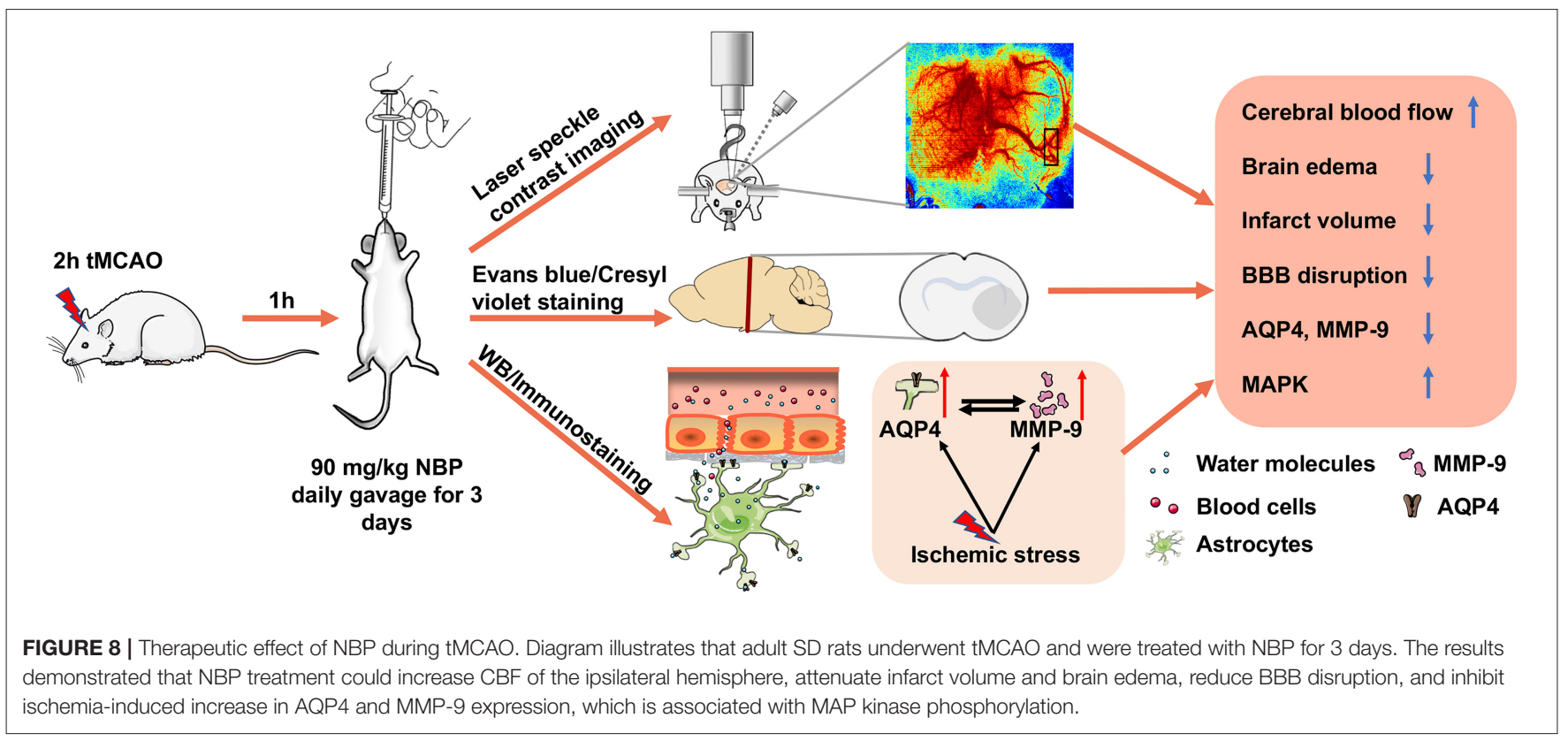




\section{CONCLUSION}

Our study demonstrated that NBP treatment increased CBF and preserved BBB integrity by inhibiting AQP4 and MMP-9 enzyme activity. MAP kinases signaling pathways are possibly associated in this mechanism.

\section{DATA AVAILABILITY STATEMENT}

The original contributions presented in the study are included in the article/supplementary materials, further inquiries can be directed to the corresponding authors.

\section{ETHICS STATEMENT}

The animal surgery and experimental protocol were approved by the Institutional Animal Care and Use Committee (IACUC), Shanghai Jiao Tong University, Shanghai, China.

\section{AUTHOR CONTRIBUTIONS}

MM participated in the research design, all experimental procedures, animal surgery, data analysis, and drafting of the first manuscript. ZW and CQ contributed to animal

\section{REFERENCES}

Abdoulaye, I. A., and Guo, Y. J. (2016). A review of recent advances in neuroprotective potential of 3-N-butylphthalide and its derivatives. Biomed. Res. Int. 2016:5012341. doi: 10.1155/2016/5012341

Cai, H., Mu, Z., Jiang, Z., Wang, Y., Yang, G.-Y., and Zhang, Z. (2015). Hypoxia-controlled matrix metalloproteinase-9 hyperexpression promotes behavioral recovery after ischemia. Neurosci. Bull. 31, 550-560. doi: 10.1007/s12264-015-1533-1

Cao, W. Y., Deji, Q. Z., Li, Q. F., He, L., and Zhou, D. (2009). Effects of dl$3 n$-butylphthalide on the expression of VEGF and bFGF in transient middle cerebral artery occlusion rats. Sichuan Da Xue Xue Bao Yi Xue Ban 40, 403-407. doi: 10.1360/972009-495

Coletta, C., Papapetropoulos, A., Erdelyi, K., Olah, G., Módis, K., Panopoulos, P., et al. (2012). Hydrogen sulfide and nitric oxide are mutually dependent in the regulation of angiogenesis and endothelium-dependent vasorelaxation. Proc. Natl. Acad. Sci. U.S.A. 109, 9161-9166. doi: 10.1073/pnas.1202916109

Feigin, V. L., Norrving, B., and Mensah, G. A. (2017). Global burden of stroke. Circ. Res. 120, 439-448. doi: 10.1161/CIRCRESAHA.116.308413

Filchenko, I., Blochet, C., Buscemi, L., Price, M., Badaut, J., and Hirt, L. (2020). Caveolin-1 regulates perivascular aquaporin-4 expression after cerebral ischemia. Front. Cell Develop. Biol. 8:371. doi: 10.3389/fcell.2020.00371

Kalogeris, T., Baines, C. P., Krenz, M., and Korthuis, R. J. (2016). Ischemia/reperfusion. Compr. Physiol. 7, 113-170. doi: 10.1002/cphy.c160006

Kao, S., Jaiswal, R. K., Kolch, W., and Landreth, G. E. (2001). Identification of the mechanisms regulating the differential activation of the mapk cascade by epidermal growth factor and nerve growth factor in PC12 cells. J. Biol. Chem. 276, 18169-18177. doi: 10.1074/jbc.M008870200

Khatri, R., McKinney, A. M., Swenson, B., and Janardhan, V. (2012). Blood-brain barrier, reperfusion injury, and hemorrhagic transformation in acute ischemic stroke. Neurology 79, 52-57. doi: 10.1212/WNL.0b013e3182697e70

Kleffner, I., Bungeroth, M., Schiffbauer, H., Schäbitz, W.-R., Ringelstein, E. B., and Kuhlenbäumer, G. (2008). The role of aquaporin-4 polymorphisms in the development of brain edema after middle cerebral artery occlusion. Stroke 39, 1333-1335. doi: 10.1161/STROKEAHA.107.500785 surgery, behavioral tests, and data collection. YT provided technical assistance for in vivo and in vitro experiments. $\mathrm{H}-\mathrm{LT}$ and YW were involved in discussion of the research design, the results, and edited the manuscript. G-YY and ZZ supervised all aspects including research design, data analysis, and manuscript preparation. All authors read and agreed to the final manuscript.

\section{FUNDING}

This study was supported by grants from the National Key Research and Development Program of China (2016YFC1300600), scientific research and innovation program of Shanghai Education Commission (2019-01-07-00-02-E00064, G-YY), the National Natural Science Foundation of China (81771244, ZZ; 81771251, G-YY; 81974179, ZZ; 81771281, F-xS; 81801170, YT; 81870921, YW), and the K. C. Wong Education Foundation (G-YY).

\section{ACKNOWLEDGMENTS}

We thank Peng Miao and Bing Bo for assisting the laser speckle contrast imaging data analysis and Shijiazhuang Pharmaceutical Group Co. Ltd. for providing NBP.
Lakhan, S., Kirchgessner, A., Tepper, D., and Aidan, L. (2013). Matrix metalloproteinases and blood-brain barrier disruption in acute ischemic stroke. Front. Neurol. 4:32. doi: 10.3389/fneur.2013.00032

Lee, J. H., Cui, H. S., Shin, S. K., Kim, J. M., Kim, S. Y., Lee, J. E., et al. (2013). Effect of propofol post-treatment on blood-brain barrier integrity and cerebral edema after transient cerebral ischemia in rats. Neurochem. Res. 38, 2276-2286. doi: 10.1007/s11064-013-1136-7

Li, J., Li, Y., Ogle, M., Zhou, X., Song, M., Yu, S. P., et al. (2010). DL-3-n-butylphthalide prevents neuronal cell death after focal cerebral ischemia in mice via the JNK pathway. Brain Res. 1359, 216-226. doi: 10.1016/j.brainres.2010.08.061

Li, Q., Tang, G., Xue, S., He, X., Miao, P., Li, Y., et al. (2013). Silica-coated superparamagnetic iron oxide nanoparticles targeting of EPCs in ischemic brain injury. Biomaterials 34, 4982-4992. doi: 10.1016/j.biomaterials.2013.03.030

Li, Y., Chopp, M., Chen, J., Wang, L., Gautam, S. C., Xu, Y.-X., et al. (2000). Intrastriatal transplantation of bone marrow nonhematopoietic cells improves functional recovery after stroke in adult mice. J. Cereb. Blood Flow Metabol. 20, 1311-1319. doi: 10.1097/00004647-200009000-00006

Liao, S. J., Lin, J. W., Pei, Z., Liu, C. L., Zeng, J. S., and Huang, R. X. (2009). Enhanced angiogenesis with dl-3n-butylphthalide treatment after focal cerebral ischemia in RHRSP. Brain Res. 1289, 69-78. doi: 10.1016/j.brainres.2009.06.018

Lin, X., Miao, P., Wang, J., Yuan, F., Guan, Y., Tang, Y., et al. (2013). Surgery-related thrombosis critically affects the brain infarct volume in mice following transient middle cerebral artery occlusion. PLOS ONE 8:e75561. doi: 10.1371/journal.pone.0075561

Liu, C. L., Liao, S. J., Zeng, J. S., Lin, J. W., Li, C. X., Xie, L. C., et al. (2007). dl$3 n$-butylphthalide prevents stroke via improvement of cerebral microvessels in RHRSP. J. Neurol. Sci. 260, 106-113. doi: 10.1016/j.jns.2007.04.025

Mamtilahun, M., Tang, G., Zhang, Z., Wang, Y., Tang, Y., and Yang, G.-Y. (2019). Targeting water in the brain: role of aquaporin-4 in ischemic brain edema. Curr. Drug Targets 20, 748-755. doi: 10.2174/1389450120666190214115309

Miao, P., Li, N., Thakor, N. V., and Tong, S. (2010). Random process estimator for laser speckle imaging of cerebral blood flow. Opt. Express. 18, 218-236. doi: 10.1364/OE.18.000218 
Obermeier, B., Daneman, R., and Ransohoff, R. M. (2013). Development, maintenance and disruption of the blood-brain barrier. Nat. Med. 19, 1584-1596. doi: 10.1038/nm.3407

Qin, C., Zhou, P., Wang, L., Mamtilahun, M., Li, W., Zhang, Z., et al. (2019). Dl3-N-butylphthalide attenuates ischemic reperfusion injury by improving the function of cerebral artery and circulation. J. Cereb. Blood Flow Metab. 39, 2011-2021. doi: 10.1177/0271678X18776833

Ribeiro, M. d. C., Hirt, L., Bogousslavsky, J., Regli, L., and Badaut, J. (2006). Time course of aquaporin expression after transient focal cerebral ischemia in mice. J. Neurosci. Res. 83, 1231-1240. doi: 10.1002/jnr.20819

Rincon-Heredia, R., Flores-Benitez, D., Flores-Maldonado, C., BonillaDelgado, J., García-Hernández, V., Verdejo-Torres, O., et al. (2014). Ouabain induces endocytosis and degradation of tight junction proteins through ERK1/2-dependent pathways. Exp. Cell Res. 320, 108-118. doi: 10.1016/j.yexcr.2013.10.008

Rosell, A., Cuadrado, E., Ortega-Aznar, A., Hernández-Guillamon, M., Lo Eng, H., and Montaner, J. (2008). MMP-9-positive neutrophil infiltration is associated to blood-brain barrier breakdown and basal lamina type IV collagen degradation during hemorrhagic transformation after human ischemic stroke. Stroke 39, 1121-1126. doi: 10.1161/STROKEAHA.107.500868

Rosenberg, G. A. (1999). Ischemic brain edema. Prog. Cardiovasc. Dis. 42, 209-216. doi: 10.1016/S0033-0620(99)70003-4

Sandoval, K. E., and Witt, K. A. (2008). Blood-brain barrier tight junction permeability and ischemic stroke. Neurobiol. Dis. 32, 200-219. doi: 10.1016/j.nbd.2008.08.005

Shi, Y., Leak, R. K., Keep, R. F., and Chen, J. (2016). Translational stroke research on blood-brain barrier damage: challenges, perspectives, and goals. Transl. Stroke Res. 7, 89-92. doi: 10.1007/s12975-016-0447-9

Stamatovic, S. M., Johnson, A. M., Sladojevic, N., Keep, R. F., and Andjelkovic, A. V. (2017). Endocytosis of tight junction proteins and the regulation of degradation and recycling. Ann. N. Y. Acad. Sci. 1397, 54-65. doi: $10.1111 /$ nyas. 13346

Sun, J., and Nan, G. (2016). The mitogen-activated protein kinase (MAPK) signaling pathway as a discovery target in stroke. J. Mol. Neurosci. 59, 90-98. doi: 10.1007/s12031-016-0717-8

Tang, G., Liu, Y., Zhang, Z., Lu, Y., Wang, Y., Huang, J., et al. (2014). Mesenchymal stem cells maintain blood-brain barrier integrity by inhibiting aquaporin-4 upregulation after cerebral ischemia. Stem Cells 32, 3150-3162. doi: $10.1002 /$ stem. 1808

Tang, S. C., Luo, C. J., Zhang, K. H., Li, K., Fan, X. H., Ning, L. P., et al. (2017). Effects of dl-3-n-butylphthalide on serum VEGF and bFGF levels in acute cerebral infarction. Eur. Rev. Med. Pharmacol. Sci. 21, 4431-4436.

Tanno, H., Nockels, R. P., Pitts, L. H., and Noble, L. J. (1992). Breakdown of the blood-brain barrier after fluid percussive brain injury in the rat. Part 1: distribution and time course of protein extravasation. J. Neurotrauma 9, 21-32. doi: 10.1089/neu.1992.9.21

Turner, R. J., and Sharp, F. R. (2016). Implications of MMP9 for blood brain barrier disruption and hemorrhagic transformation following ischemic stroke. Front. Cell. Neurosci. 10:56. doi: 10.3389/fncel.2016.00056

Wang, J., Lin, X., Mu, Z., Shen, F., Zhang, L., Xie, Q., et al. (2019). Rapamycin increases collateral circulation in rodent brain after focal ischemia as detected by multiple modality dynamic imaging. Theranostics 9, 4923-4934. doi: $10.7150 /$ thno. 32676

Wang, S., Ma, F., Huang, L., Zhang, Y., Peng, Y., Xing, C., et al. (2018). Dl-3-n-butylphthalide (NBP): a promising therapeutic agent for ischemic stroke. CNS Neurol. Disord. Drug Targets 17, 338-347. doi: 10.2174/1871527317666180612125843

Wang, X., Li, Y., Zhao, Q., Min, Z., Zhang, C., Lai, Y., et al. (2011). Design, synthesis and evaluation of nitric oxide releasing derivatives of 3-nbutylphthalide as antiplatelet and antithrombotic agents. Org. Biomol. Chem. 9, 5670-5681. doi: 10.1039/c1ob05478c
Wang, X.-L., Wang, Z.-Y., Ling, J.-J., Zhang, Y.-H., and Yin, J. (2016). Synthesis and biological evaluation of nitric oxide (NO)-hydrogen sulfide $(\mathrm{H}(2) \mathrm{S})$ releasing derivatives of $(\mathrm{S})-3-\mathrm{n}$-butylphthalide as potential antiplatelet agents. Chin. J. Nat. Med. 14, 946-953. doi: 10.1016/S1875-5364(17)30 021-3

Wang, Z., Meng, C.-J., Shen, X.-M., Shu, Z., Ma, C., Zhu, G.-Q., et al. (2012). Potential contribution of hypoxia-inducible factor- $1 \alpha$, aquaporin-4, and matrix metalloproteinase- 9 to blood-brain barrier disruption and brain edema after experimental subarachnoid hemorrhage. J. Mol. Neurosci. 48, 273-280. doi: 10.1007/s12031-012-9769-6

Writing Group, M., Mozaffarian, D., Benjamin, E. J., Go, A. S., Arnett, D. K., Blaha, M. J., et al. (2016). Heart disease and stroke statistics-2016 update: a report from the american heart association. Circulation 133, e38-e360. doi: 10.1161/CIR.0000000000000350

Yang, C.-S., Guo, A., Li, Y., Shi, K., Shi, F.-D., and Li, M. (2019). Dl-3-nbutylphthalide reduces neurovascular inflammation and ischemic brain injury in mice. Aging Dis. 10, 964-976. doi: 10.14336/AD.2019.0608

Yang, Y., Estrada, E. Y., Thompson, J. F., Liu, W., and Rosenberg, G. A. (2007). Matrix metalloproteinase-mediated disruption of tight junction proteins in cerebral vessels is reversed by synthetic matrix metalloproteinase inhibitor in focal ischemia in rat. J. Cereb. Blood Flow Metab. 27, 697-709. doi: 10.1038/sj.jcbfm.9600375

Yang, Y., and Rosenberg Gary, A. (2011). Blood-brain barrier breakdown in acute and chronic cerebrovascular disease. Stroke 42, 3323-3328. doi: 10.1161/STROKEAHA.110.608257

Ye, Z. Y., Xing, H. Y., Wang, B., Liu, M., and Lv, P. Y. (2019). DL-3-nbutylphthalide protects the blood-brain barrier against ischemia/hypoxia injury via upregulation of tight junction proteins. Chin. Med. J. 132, 1344-1353. doi: 10.1097/CM9.0000000000000232

Zhang, C., Zhao, S., Zang, Y., Gu, F., Mao, S., Feng, S., et al. (2017) The efficacy and safety of Dl-3n-butylphthalide on progressive cerebral infarction: a randomized controlled STROBE study. Medicine 96:e7257. doi: 10.1097/MD.0000000000007257

Zhang, L., Lü, L., Chan, W. M., Huang, Y., Wai, M. S., and Yew, D. T. (2012a). Effects of DL-3-n-butylphthalide on vascular dementia and angiogenesis. Neurochem. Res. 37, 911-919. doi: 10.1007/s11064-011-0663-3

Zhang, L., Yu, W. H., Wang, Y. X., Wang, C., Zhao, F., Qi, W., et al. (2012b). DL3-n-Butylphthalide, an anti-oxidant agent, prevents neurological deficits and cerebral injury following stroke per functional analysis, magnetic resonance imaging and histological assessment. Curr. Neurovasc. Res. 9, 167-175. doi: $10.2174 / 156720212801618956$

Zhang, T., Jia, W., and Sun, X. (2010). 3-n-Butylphthalide (NBP) reduces apoptosis and enhances vascular endothelial growth factor (VEGF) up-regulation in diabetic rats. Neurol. Res. 32, 390-396. doi: 10.1179/016164110X12670144526264

Zhou, P.-T., Wang, L.-P., Qu, M.-J., Shen, H., Zheng, H.-R., Deng, L.-D., et al. (2019). Dl-3-N-butylphthalide promotes angiogenesis and upregulates sonic hedgehog expression after cerebral ischemia in rats. CNS Neurosci. Therap. 25, 748-758. doi: $10.1111 /$ cns.13104

Conflict of Interest: The authors declare that the research was conducted in the absence of any commercial or financial relationships that could be construed as a potential conflict of interest.

Copyright (C) 2021 Mamtilahun, Wei, Qin, Wang, Tang, Shen, Tian, Zhang and Yang. This is an open-access article distributed under the terms of the Creative Commons Attribution License (CC BY). The use, distribution or reproduction in other forums is permitted, provided the original author(s) and the copyright owner(s) are credited and that the original publication in this journal is cited, in accordance with accepted academic practice. No use, distribution or reproduction is permitted which does not comply with these terms. 